\title{
Acceleration training for managing nonalcoholic fatty liver disease: a pilot study
}

This article was published in the following Dove Press journal:

Therapeutics and Clinical Risk Management

7 November 2014

Number of times this article has been viewed

\author{
Sechang $\mathrm{Oh}^{1}$ \\ Takashi Shida' \\ Akemi Sawai' \\ Tsuyoshi Maruyama ${ }^{2}$ \\ Kiyoshi Eguchi \\ Tomonori Isobe' \\ Yoshikazu Okamoto ${ }^{3}$ \\ Noriko Someya ${ }^{4}$ \\ Kiyoji Tanaka ${ }^{4}$ \\ Emi Arai' \\ Akiko Tozawa ${ }^{5}$ \\ Junichi Shoda \\ 'Department of Medical Sciences, \\ Faculty of Medicine, University \\ of Tsukuba, ${ }^{2}$ Department of \\ Rehabilitation, Tsukuba University \\ Hospital, ${ }^{3}$ Department of Diagnostic \\ Radiology, ${ }^{4}$ Department of Sports \\ Medicine, Faculty of Health and Sport \\ Sciences, University of Tsukuba, \\ Ibaraki, ${ }^{5}$ Protea Japan Co Ltd, Chiyoda, \\ Tokyo, Japan
}

Background: While aerobic training is generally recommended as therapeutic exercise in guidelines, the effectiveness of resistance training has recently been reported in the management of nonalcoholic fatty liver disease (NAFLD). Acceleration training (AT) is a new training method that provides a physical stimulation effect on skeletal muscles by increasing gravitational acceleration with vibration. AT has recently been indicated as a component of medicine. In this study, we evaluated the effectiveness of AT in the management of NAFLD in obese subjects. Methods: A total of 18 obese patients with NAFLD who had no improvement in liver function test abnormalities and/or steatosis grade after 12 weeks of lifestyle counseling were enrolled in an AT program. These patients attended a 20-minute session of AT twice a week for 12 consecutive weeks.

Results: During the AT program, the NAFLD patients showed a modest increase in the strength $(+12.6 \%)$ and cross-sectional area $(+3.1 \%)$ of the quadriceps, coupled with a significant reduction in intramyocellular lipids $(-26.4 \%)$. Notably, they showed a modest reduction in body weight $(-1.9 \%)$, abdominal visceral fat area $(-3.4 \%)$, and hepatic fat content $(-8.7 \%)$, coupled with a significant reduction in levels of aminotransferase $(-15.7 \%), \gamma$-glutamyltransferase $(-14.4 \%)$, leptin $(-9.7 \%)$, interleukin-6 (-26.8\%), and tumor necrosis factor- $\alpha(-17.9 \%)$, and a significant increase of adiponectin $(+8.7 \%)$. On a health-related quality of life survey, the patients showed an improvement in physical functioning $(+17.3 \%)$, physical role $(+9.7 \%)$, general health $(+22.1)$, and social functioning $(+6.0 \%)$.

Conclusion: AT reduced hepatic and intramyocellular fat contents and ameliorated liver function test abnormalities in obese patients with NAFLD, which was coupled with improved physical function and body adiposity. AT is clinically beneficial for the management of NAFLD.

Keywords: whole-body vibration, obesity, liver steatosis, adipokine, quality of life

\section{Introduction}

In Japan, national obesity trends have been adversely affected by westernized dietary habits and chronic physical inactivity. The prevalence of obesity among the adult population is about $30 \%$, and has increased by about 1.5 -fold over the last 30 years among men. ${ }^{1}$ Consequently, the incidences of lifestyle disease-associated conditions such as hypertension, high cholesterol, and impaired glucose tolerance are steadily increasing. ${ }^{2}$ In particular, the occurrence of abnormal hepatic function is especially high, with the underlying cause possibly being an increase in the prevalence of nonalcoholic fatty liver disease (NAFLD). ${ }^{2}$ NAFLD is a chronic disease characterized by accumulation of fat deposits in the liver and is considered to be the hepatic phenotype of metabolic syndrome. ${ }^{3}$

To date, no evidence-based therapy has proven to be effective for NAFLD except exercise and dietary interventions. ${ }^{4}$ Thus, aerobic exercise for at least 20 minutes a day is recommended for NAFLD management. ${ }^{5}$ However, obese people have an extra
Correspondence: Junichi Shoda Department of Medical Sciences, Faculty of Medicine, University of Tsukuba, Tsukuba, Ibaraki 305-8575, Japan

Tel +8 I 298535795

Fax +8I 298535795

Email shodaj@md.tsukuba.ac.jp
Therapeutics and Clinical Risk Management 20I4:I0 925-936
cC) (i) (c) 2014 oh et al. This work is published by Dove Medical Press Limited, and licensed under Creative Commons Attribution - Non Commercial (unported, v3.0) License. The full terms of the License are available at http://creativecommons.org/licenses/by-nc/3.0/. Non-commercial uses of the work are permitted without any further permission from Dove Medical Press Limited, provided the work is properly attributed. Permissions beyond the scope of the License are administered by Dove Medical Press Limited. Information on how to request permission may be found at: http://www.dovepress.com/permissions.php 
burden on the knee and hip joints that tends to discourage continued adherence to aerobic exercise programs. Therefore, there is an unmet need for the development of a safe and effective exercise therapy that can be sustainably adopted by NAFLD patients to achieve long-term weight loss.

In recent years, the effectiveness of resistance exercise has been suggested by a number of studies ${ }^{6,7}$ In resistance exercise, energy consumption is not very high; however, it causes an increase in muscle mass, leading to an increase in basal energy expenditure (EE) ${ }^{8,9}$ Although there is, as of yet, no evidence attesting to the long-term effect of resistance training in NAFLD specifically, resistance training is considered beneficial for the management of pathological conditions associated with obesity, especially insulin resistance. ${ }^{6}$ An exercise program of three sessions of high-intensity to moderate-intensity resistance training a week is recommended for the management of NAFLD. ${ }^{5}$

A new training method, termed whole-body vibration (WBV), has recently been introduced. This method employs equipment with a platform that vibrates at high speed and low amplitude. ${ }^{10}$ The principle behind WBV is that energy generated by the vibration device is transferred to the human body as a resonator. Mechanical oscillations of the vibration device have a sinusoidal shape, and the vibration acceleration $\left(\mathrm{m} / \mathrm{s}^{2}\right)$ and gravitational acceleration ( $\mathrm{G} ; 1 \mathrm{G}=9.81 \mathrm{~m} / \mathrm{s}^{2}$ ) are determined by adjusting frequency $(\mathrm{Hz})$ and amplitude (mm). On the WBV platform, a load corresponding to the product of body weight and gravity plus vibration acceleration is placed on the body (Figure 1). The vibration device generates three-dimensional vibrations in the vertical (z), frontal (x), and sagittal (y) directions. The acceleration in the vertical direction is considered to be the most effective component in inducing beneficial effects on the human body. Many ongoing studies are analyzing the effectiveness of WBV in subjects of different ages, health states, and physical activity levels.

Acceleration training (AT), another WBV-based approach, is a training program that involves various physical movements or still posture optimized for individual purpose and is performed on a platform that vibrates three-dimensionally. The advantage of AT is that it can provide the beneficial effects of resistance exercise without dynamic physical movements or heavy loading. Many previous studies ${ }^{11,12}$ and our recent study ${ }^{13}$ have shown that AT, as a new tool for rehabilitation, leads to improvement in lower limb function, an increase in muscle mass and strength, and a reduction of visceral abdominal fat. In addition, AT can transmit stable vibrations to the human body and has a low impact on the

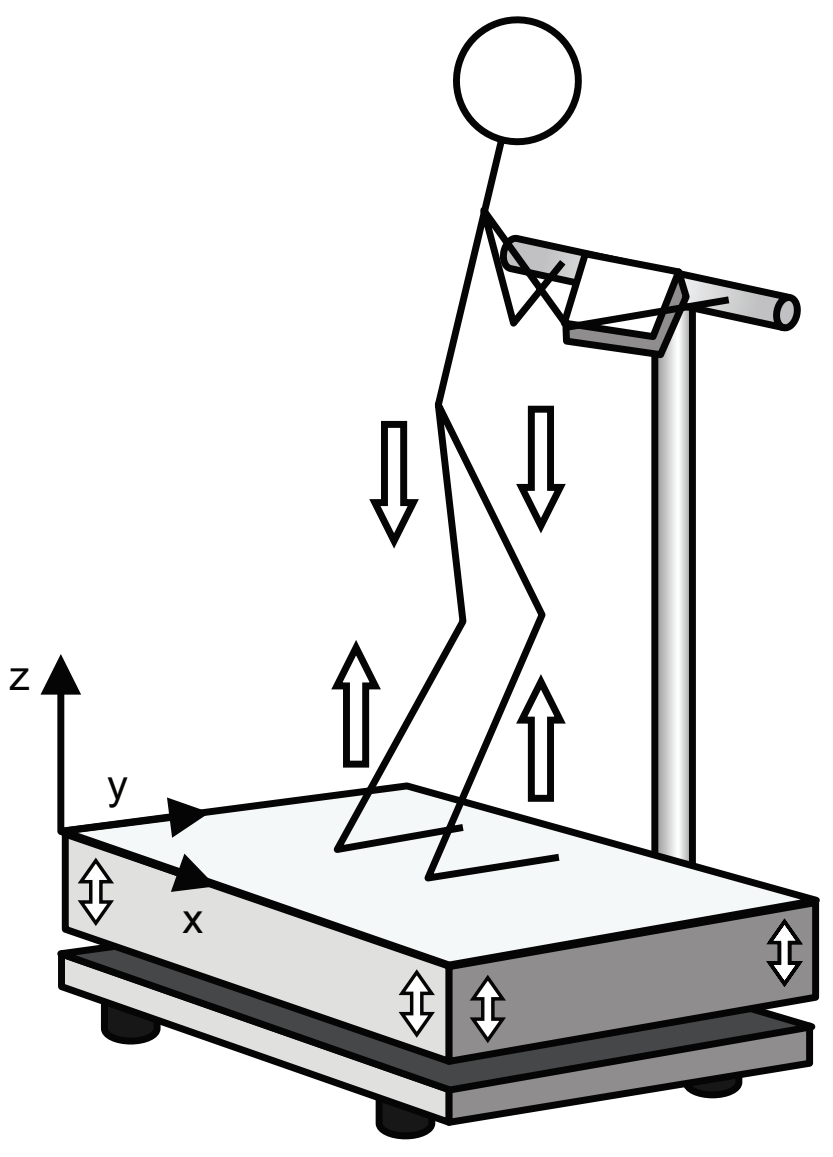

Figure I Illustration of the synchronous mode of vibration transmission in wholebody vibration exercise. Both legs extend and stretch at the same time, and a purely linear acceleration is directed to the trunk.

knee and hip joints, meaning it might be a suitable exercise approach for obese patients who have difficulties engaging in physical activities because of joint pain.

In this pilot study, we evaluated the outcome of AT in obese patients with NAFLD by analyzing physical function, body composition, hepatic and metabolic function, fat contents in the liver and skeletal muscles by imaging analysis, and the effect on quality of life with regard to physical and mental health.

\section{Materials and methods Subjects and study design}

Between April 2012 and December 2013, 66 obese subjects with NAFLD presented at the outpatient department of the University of Tsukuba Hospital. The diagnosis of NAFLD was based on overeating or physical inactivity, elevated serum alanine aminotransferase (ALT) levels, and the presence of at least two of three abnormal findings using abdominal ultrasonography: diffusely increased liver echogenicity ("bright") that was greater than for kidney, vascular blurring, 
and deep attenuation of the ultrasound signal according to the diagnostic guidelines for NAFLD in the Asia-Pacific region. ${ }^{14}$ We excluded patients with alcohol consumption $>20 \mathrm{~g} /$ day. Patients with the following conditions were also excluded from this study: presence of other causes of liver disease, psychiatric diseases, and taking anti-diabetic agents or weight loss agents. Each patient received lifestyle counseling regarding diet and physical activity for NAFLD according to the guidelines of the American Gastroenterological Association. ${ }^{15,16}$ The counseling was conducted with a registered dietitian and a medical doctor majoring in nutrition. Twelve weeks after the counseling, 48 patients showed either a decrease or no change in serum ALT levels and liver fat deposition evaluated by abdominal ultrasonography. The remaining 18 patients showed an increase in serum ALT levels and/or the grade of liver fat deposition and participated in the 12-week AT program. In addition, 19 nonobese and normolipidemic participants (normal subjects), who worked for the University of Tsukuba Hospital, were enrolled in the study and underwent examinations of body composition, proton magnetic spectroscopy, liver steatosis and stiffness, and blood analysis.

The study protocol conformed to the ethical guidelines of the 1975 Declaration of Helsinki as reflected in prior approval by the ethics committee of the School of Medicine, University of Tsukuba. Written informed consent was obtained from each patient.

\section{AT program}

Eighteen participants ( 4 men and 14 women) took part in the AT program twice a week for 12 weeks. Participants performed upper and lower body exercises on a vertical vibration machine (Power Plate Pro6, Badhoevendorp, the Netherlands). Participants performed the AT protocol consisting of three sessions (movement preparation, strength and power, and massage) for a total of 40 minutes, including a rest interval of 30 seconds after each movement. A movement preparation session included hamstring stretch, calf stretch, side stretch, and hip joint stretch (frequency, $30 \mathrm{~Hz}$; amplitude, low; time, 30 seconds; set, 2). A strength and power session included deep squat, wide stance squat, lunge, push up, triceps dips, crunch, front plank, and pelvic bridge (frequency, 30-35 Hz; amplitude, low; time, 30 seconds; set, 2). A final session consisted of massage of the calf, hamstring, lower back, shoulder, and face (frequency, $40 \mathrm{~Hz}$; amplitude, high; time, 60 seconds; set, 2). Trained staff supervised all training sessions to ensure correct execution (Figure S1 provides details on the AT program used in this study). During the AT period, participants did not receive any lifestyle counseling, and were not firmly restricted with regard to their dietary intake or regular physical activity.

\section{Assessment of energy intake and expenditure}

Participants in this pilot study maintained daily food intake records for 3 consecutive days after receiving detailed instructions at baseline and at week 12. A registered dietitian used the food intake records to estimate total daily energy intake and macronutrient composition (carbohydrate, protein, and fat intake) using Excel Eiyo-Kun version 4 software (Kenpakusya, Tokyo, Japan). Total daily EE and walking (steps/day) were measured by using a uniaxial accelerometer (Lifecorder; Suzuken Co Ltd, Nagoya, Japan). The accelerometer was firmly attached to the participant's clothing during all active hours, except while bathing and sleeping, 2 weeks before and after the study period.

\section{Indirect calorimetry}

Indirect calorimetry studies were conducted to measure ventilation and gas exchange in an open-flow indirect calorimeter (Minato Medical Science, Tokyo, Japan). Calorimetry was acquired under fasting conditions prior to and after the AT intervention. Oxygen consumption $\left(\mathrm{VO}_{2}\right)$ and carbon dioxide production $\left(\mathrm{VCO}_{2}\right)$ were measured for each chamber every 15 minutes. The respiratory exchange ratio $\left(\mathrm{RER}=\mathrm{VCO}_{2} / \mathrm{VO}_{2}\right)$ was calculated. $\mathrm{EE}$ was calculated using the equation $\mathrm{EE}=\mathrm{VO}_{2} \times[3.815+(1.232 \times \mathrm{RER})]$, and normalized for subject body mass.

\section{Body weight and composition}

Body weight and composition and abdominal visceral fat area were measured using an InBody720 (Biospace Japan Inc, Tokyo, Japan). Standing height was measured by a wallmounted stadiometer (Muratec-KDS Co, Kyoto, Japan) and used to calculate body mass index $\left(\mathrm{kg} / \mathrm{m}^{2}\right)$.

\section{Laboratory determinations}

Blood samples were drawn from the median cubital vein at baseline and at week 12 following a period of no training for 48 hours and after fasting for 12 hours. Total cholesterol, triglyceride, free fatty acid, and high-density lipoprotein cholesterol levels were analyzed enzymatically as follows: aspartate aminotransferase, ALT, and gamma glutamyl transpeptidase levels by the Japan Society of Clinical Chemistry transferable method; hyaluronic acid, ferritin, and plasma type IV collagen levels by a latex agglutination method 
(Bio Majesty JCA-BM 8060; JEOL Ltd, Tokyo, Japan); fasting plasma insulin level by a chemiluminescent immunoassay method (Modular E170; Roche Diagnostics Corporation, Indianapolis, IN, USA); fasting plasma glucose levels by a hexokinase-G-6-PDH method, and glycated hemoglobin $\left(\mathrm{HbA}_{1 \mathrm{c}}\right)$ by a latex agglutination method (JCA-BM 9130); and high-sensitivity C-reactive protein level by a fixed time assay method (Behring Nephelometer II; Siemens Healthcare Diagnostics, Marburg, Germany). Commercial enzyme-linked immunosorbent assay kits were used to measure serum levels of total adiponectin (Sekisui Medical Co Ltd, Tokyo, Japan), tumor necrosis factor-alpha (TNF- $\alpha$ ), interleukin-6, leptin (R\&D Systems Inc, Minneapolis, MN, USA), and thiobarbituric acid reactive substances (Cayman Chemical Co, Ann Arbor, MI, USA). Surrogate markers were calculated from insulin resistance by homeostasis model assessment. ${ }^{17}$

\section{Ultrasound}

An SSD-5500 ultrasound scanner (Aloka Co Ltd, Tokyo, Japan) was used to evaluate liver steatosis. The grade of liver steatosis was decided by a clinical radiologist according to the criteria of Saadeh et al. ${ }^{16}$ The radiologist was blinded to the treatment allocation of the patients. The films were scored in random order without any clinical information.

\section{Assessment of hepatic stiffness and steatosis}

A clinical gastroenterologist assessed hepatic stiffness using a Fibroscan (Echosens, Paris, France) with a $3.5 \mathrm{MHz}$ standard probe. The principle and examination procedures have been published previously. ${ }^{18}$ Additionally, for subjects who participated in this program in 2012, we determined the quantity of hepatic lipids with a controlled attenuation parameter (CAP) designed to measure liver ultrasonic attenuation (go and return path) at $3.5 \mathrm{MHz}$ by using signals acquired by the Fibroscan. Detailed descriptions of CAP have also been published previously. ${ }^{19}$

\section{Magnetic resonance spectroscopy}

The tissue fat content in the liver and muscles was determined using proton magnetic resonance spectroscopy ( $\left.{ }^{1} \mathrm{H}-\mathrm{MRS}\right)$. A 3-Tesla magnetic resonance device (Achieva; Philips Electronics Japan Ltd, Tokyo, Japan) equipped with a 6-channel TORSO coil was used for the analysis. Intramyocellular lipids (IMCL) and extramyocellular lipids (EMCL) in the bilateral quadriceps were determined by the excitation method that was spin echo point-resolved spatially localized spectroscopy, and the data were acquired by the single voxel method.
The obtained data were analyzed with LCModel software (LA Systems Inc, Tokyo, Japan). The IMCL and EMCL data were given as the averages of the right and left muscles. The cross-sections of quadriceps and those of hamstrings were measured by magnetic resonance imaging. Moreover, data acquisition of ${ }^{1} \mathrm{H}-\mathrm{MRS}$ for the liver was conducted in the same way as that for measurement of IMCL and EMCL. Intrahepatic lipids were determined by calculating the ratio of the intrahepatic fat signal to water signal (lipids/water).

\section{Measurement of muscle strength}

The maximal strength of isometric contraction of knee flexion and extension at an angle of $90^{\circ}$ in a seated position was measured using a dynamometer Biodex III (Biodex Medical Systems Inc, Shirley, NY, USA). The peak torque (Nm) of the records of three consecutive trials was considered as the maximal strength. Each isometric contraction was held for $3 \mathrm{sec}-$ onds. The results were normalized by body weight $(\mathrm{kg})$.

\section{Assessment of health-related quality of life}

Health-related quality of life was assessed by a Japanesevalidated version of the Short Form Health Survey (SF-36) prior to and after the intervention. The subscales physical functioning, physical role, bodily pain, general health, vitality, social functioning, emotional role, and mental health were calculated from the questionnaires as described previously. ${ }^{20}$ Physical and mental summary measurements were obtained from the sum scores of the subscales.

\section{Statistical analysis}

The statistical analysis was performed using Statistical Package for the Social Sciences version 20.0 software (SPSS Inc, Chicago, IL, USA). Descriptive statistics were presented as the median (95\% confidence interval). The effects of AT were evaluated by changes in the variable and analyzed using the Wilcoxon signed-rank test. Differences between the groups (normal subject versus AT group pre and post) were analyzed using one-way ranked analysis of variance. A $P$-value of $<0.05$ was defined to be statistically significant.

\section{Results}

\section{Energy expenditure and energy intake}

The participants showed no significant changes in resting EE $(-0.7 \%)$ or nonprotein respiratory quotient $(+1.23 \%)$ after the 12-week AT program (Table 1). According to the records of the Lifecorder, there were no significant differences in total daily EE in daily life (-14.0\%) during the time periods before and after the AT program. Moreover, according to the daily 
Table I Intervention outcomes for energy expenditure and intake, and anthropometry in obese patients with nonalcoholic fatty liver disease

\begin{tabular}{|c|c|c|c|c|}
\hline Parameter & Baseline & After 12 weeks & Change & $P$-value \\
\hline \multicolumn{5}{|c|}{ Energy expenditure and intake } \\
\hline Resting EE (kcal/day) & I,485 (I,3|7-I,726) & I,474 (I,329-I,709) & -11 & 0.96 \\
\hline npRQ & $0.81(0.75-0.91)$ & $0.82(0.78-0.86)$ & +0.01 & 0.73 \\
\hline TEE (kcal/day) & $2,292(1,825-2,645)$ & $1,972(1,862-2,513)$ & -320 & 0.11 \\
\hline TEl (kcal/day) & $1,873(1,5 \mid 2-2,760)$ & $2,109(1,458-2,880)$ & +236 & 0.21 \\
\hline
\end{tabular}

Notes: Values are presented as the median ( $95 \%$ confidence interval). The comparison of within-group changes over time, from baseline to after 12 weeks, for all dependent variables was analyzed by Wilcoxon's rank sum test.

Abbreviations: npRQ, nonprotein respiratory quotient; EE, energy expenditure; TEE, total daily energy expenditure; TEl, total daily energy intake.

food intake records, the participants did not significantly change their quantity of total daily energy intake $(+12.6 \%)$ after the AT program (Table 1).

\section{Anthropometry}

The participants showed a modest but significant reduction in body weight $(-0.4 \%)$, body mass index $(-0.7 \%)$, body fat mass $(-5.9 \%)$, and visceral fat area $(-6.8 \%)$ after the 12 -week AT program $(P<0.05)$. No significant change was found in skeletal muscle mass $(+2.0 \%)$, as shown in Table 2. However, these parameters at both baseline and after 12 weeks of AT intervention, except for skeletal muscle mass, were significantly higher than that in normal subjects $(P<0.01)$.

\section{Muscle strength and cross-sectional area}

The participants underwent muscle strength testing before and after the AT program (Figure 2A). A significant increase was observed in median values for strength

Table 2 Intervention adherence outcomes for blood biochemistry in obese patients with nonalcoholic fatty liver disease

\begin{tabular}{|c|c|c|c|c|c|}
\hline \multirow[t]{2}{*}{ Parameter } & \multirow{2}{*}{$\frac{\text { Normal subjects }}{\text { Pre }}$} & \multicolumn{4}{|l|}{ AT } \\
\hline & & Pre & Post & Change & P-value \\
\hline \multicolumn{6}{|l|}{ Anthropometry } \\
\hline Body weight (kg) & $53.3(47.6-63.8)$ & $73.8(66.8-96.6)^{\dagger \dagger}$ & $73.5(65.5-91.6)^{\dagger \dagger}$ & -0.3 & $<0.05$ \\
\hline BMI $\left(\mathrm{kg} / \mathrm{m}^{2}\right)$ & $20.3(19.0-21.8)$ & $28.2(25.5-33.2)^{\dagger \dagger}$ & $28.0(25.3-33.3)^{\dagger \dagger}$ & -0.2 & $<0.05$ \\
\hline Body fat mass $(\mathrm{kg})$ & I I.0 (7.9-| 5.1$)$ & $28.9(22.3-38.2)^{\dagger \dagger}$ & $27.2(18.4-37.8)^{\dagger \dagger}$ & -1.7 & $<0.05$ \\
\hline Skeletal muscle (kg) & $23.3(19.1-28.1)$ & $24.5(2 I . I-30.0)$ & $25.0(21.4-29.2)$ & +0.5 & 0.68 \\
\hline Visceral fat area $\left(\mathrm{cm}^{2}\right)$ & 42.5 (31.9-49.0) & $132.0(106.6-143.7)^{\dagger \dagger}$ & $123.0(106.2-146.4)^{\dagger \dagger}$ & -9.0 & $<0.05$ \\
\hline \multicolumn{6}{|l|}{ Blood analysis } \\
\hline AST (U/L) & $16.0(15.0-19.0)$ & $25.0(19.0-41.0)^{\dagger \dagger}$ & $21.0(18.0-34.0)^{\dagger+}$ & -11.7 & 0.29 \\
\hline ALT (U/L) & |3.0 (| I.0-|7.0) & $33.0(20.0-53.0)^{\dagger \dagger}$ & $22.5(18.0-43.0)^{\dagger \dagger}$ & -15.7 & $<0.05$ \\
\hline$\gamma$-GT $(\mathrm{U} / \mathrm{L})$ & I5.0 (I2.0-23.0) & $28.0(24.0-38.0)^{\dagger \dagger}$ & $26.0(19.0-37.0)^{\dagger \dagger}$ & -2.0 & $<0.05$ \\
\hline Ferritin (ng/mL) & $61.3(20.8-125.0)$ & $74.9(26.8-123.0)^{\dagger}$ & $60.8(29.8-96.9)$ & -14.1 & 0.34 \\
\hline hs-CRP (mg/dL) & $0.01(0.01-0.02)$ & $0.16(0.05-0.28)^{\dagger}$ & $0.06(0.03-0.33)$ & -0.10 & 0.27 \\
\hline Platelet $\left(10^{4} / \mu \mathrm{L}\right)$ & $23.3(21.9-29.7)$ & $24.8(15.4-29.8)$ & $25.4(17.6-29.5)$ & +0.6 & 0.24 \\
\hline FFA (mEq/L) & $0.45(0.37-0.60)$ & $0.61(0.49-0.74)^{\dagger}$ & $0.5 \mathrm{I}(0.4 \mathrm{I}-0.56)$ & -0.10 & $<0.05$ \\
\hline Triglycerides (mg/dL) & $86.0(42.0-100.0)$ & II $5.0(85.0-\mid 64.0)^{\dagger}$ & II $0.0(83.0-165.0)^{\dagger}$ & -5.0 & 0.76 \\
\hline Total cholesterol (mg/dL) & $188.0(166.0-195.0)$ & 195.5 (I78.0-223.0) & $185.5(165.0-210.0)$ & -10.0 & $<0.05$ \\
\hline HDL-C (mg/dL) & $60.0(55.0-73.0)$ & $51.8(46.9-63.3)^{\dagger}$ & $53.1(47.3-59.3)^{\dagger}$ & $+\mathrm{I} .3$ & 0.86 \\
\hline LDL-C (mg/dL) & $106.0(87.0-132.0)$ & I I0.0 (90.0-127.0) & $102.0(93.0-123.0)$ & -8.0 & 0.08 \\
\hline $\mathrm{FPG}(\mathrm{mg} / \mathrm{dL})$ & $77.0(73.0-85.0)$ & $94.5(88.0-102.0)^{\dagger \dagger}$ & $99.0(89.0-107.0)^{\dagger \dagger}$ & +4.5 & 0.40 \\
\hline $\mathrm{FPI}(\mu \mathrm{U} / \mathrm{mL})$ & $4.6(2.80-6.60)$ & $8.3(5.50-11.8)^{\dagger \dagger}$ & $10.8(4.30-12.6)^{\dagger \dagger}$ & +2.5 & 0.85 \\
\hline $\mathrm{HbA}_{\mathrm{Ic}}(\%)$ & $4.8(4.70-4.90)$ & $5.2(4.90-5.80)^{\dagger \dagger}$ & $5.3(4.90-5.50)^{\dagger \dagger}$ & +0.1 & 0.81 \\
\hline HOMA-IR & $0.9(0.53-1.25)$ & $2.0(1.20-3.26)^{t \dagger}$ & $2.3(1.06-3.26)^{\dagger \dagger}$ & +0.3 & 0.75 \\
\hline Hyaluronan (ng/mL) & I4.5 (I0.I-20.7) & $31.5(10.0-43.0)$ & $24.0(10.0-48.0)$ & -7.5 & 0.46 \\
\hline Type IV collagen (ng/mL) & $104.0(90.0-117.0)$ & | $19.0(99.0-142.0)^{\dagger}$ & $123.1(103.0-148.0)^{\dagger}$ & +4.1 & 0.60 \\
\hline
\end{tabular}

Notes: Values are presented as the median ( $95 \%$ confidence interval). The comparison of within-group changes over time, from baseline to after 12 weeks, for all dependent variables was analyzed by Wilcoxon's rank sum test. ${ }^{t} P<0.05$, ${ }^{t} P<0.0$ I, significant difference between groups (normal group versus acceleration training group pre and post). Abbreviations: AT, acceleration training; BMI, body mass index; AST, aspartate aminotransferase; ALT, alanine aminotransferase; $\gamma$-GT, gamma glutamyl transpeptidase; hs-CRP, high-sensitivity C-reactive protein; FFA, free fatty acids; HDL-C, high density lipoprotein cholesterol; LDL-C, low density lipoprotein cholesterol; FPG, fasting plasma glucose; FPI, fasting plasma insulin; $\mathrm{HbA}_{\mathrm{Ic}}$, glycated hemoglobin; HOMA-IR, insulin resistance by homeostasis model assessment. 
A

Quadriceps

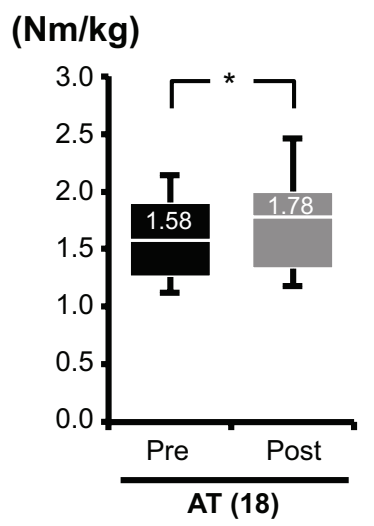

C

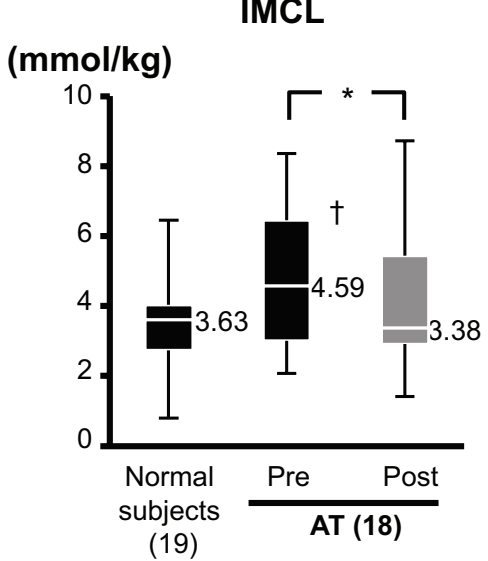

Hamstrings

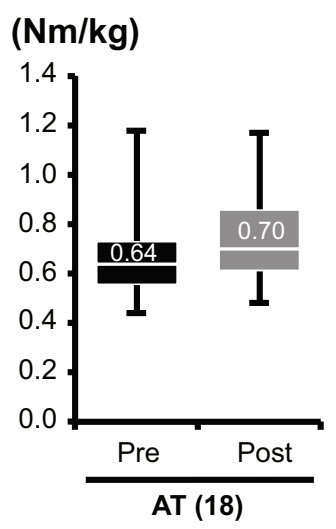

B Quadriceps



Hamstrings

$\left(\mathrm{cm}^{2}\right)$

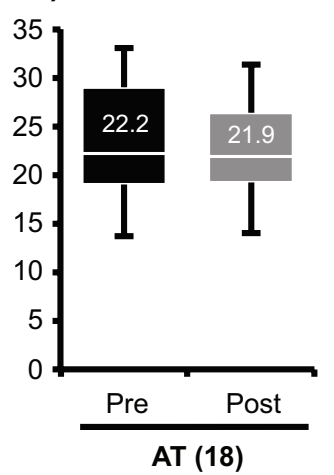

C



D

IHL
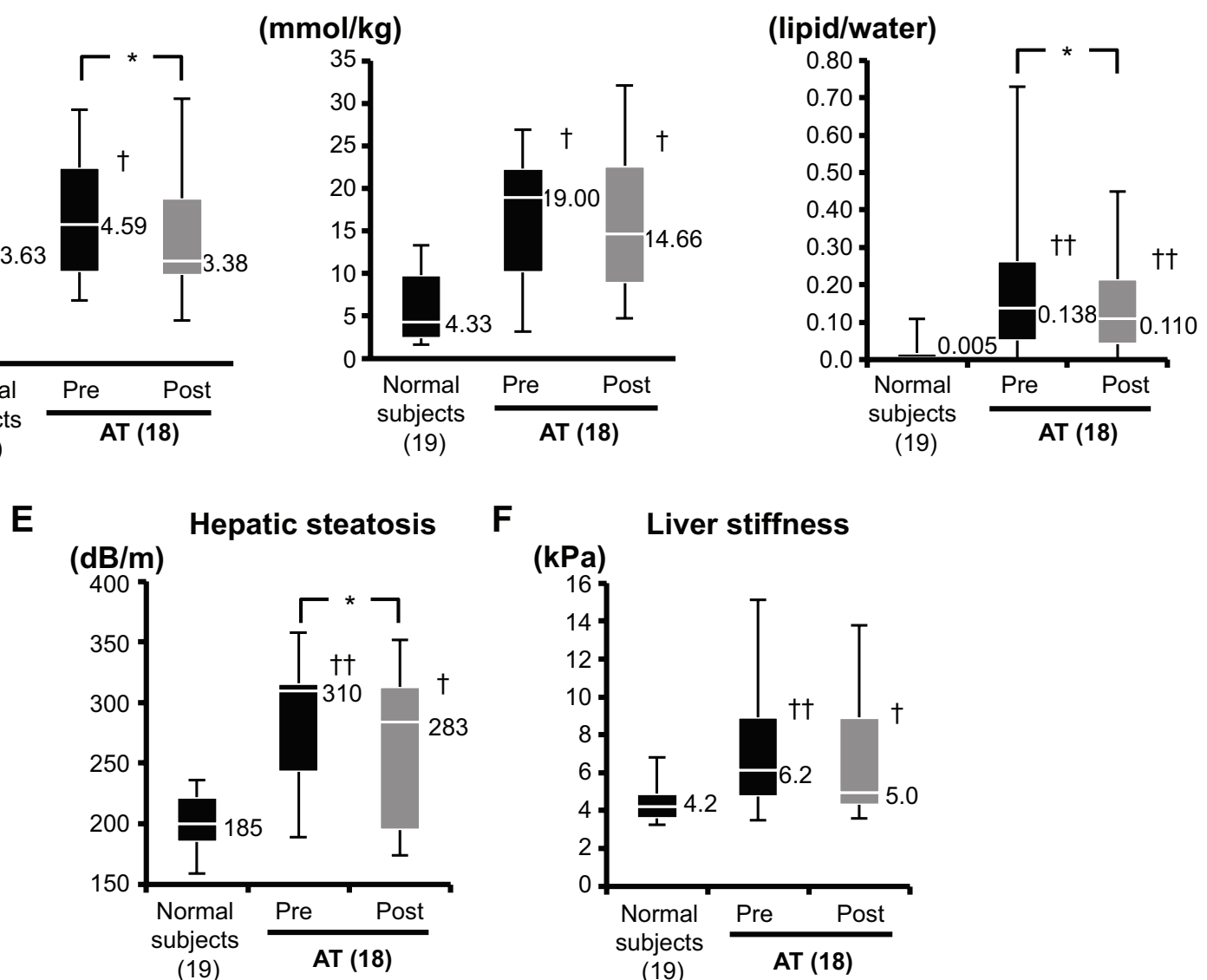

Figure 2 Effects of AT on muscle strength (A), cross-sectional area (B), fat deposition (C) in the femoral muscle (quadriceps), intrahepatocellular lipids measured by magnetic resonance spectroscopy (D), intrahepatocellular lipids measured by Fibroscan 502 (E), and liver stiffness (F) in obese patients with nonalcoholic fatty liver disease.

Notes: ${ }^{*} P<0.05$, significant difference versus baseline and week 12 . ${ }^{\dagger} P 0.05,{ }^{\dagger} P<0.01$, significant difference between groups (normal group versus AT group pre and post). The lower edge, midline, and upper edge of each box represent the 25 th percentile, median, and 75 th percentile scores, respectively. The line from each box extends to the minimum and maximum score.

Abbreviations: AT, acceleration training; IMCL, intramyocellular lipids; EMCL, extramyocellular lipids; IHL, intrahepatic lipids.

in the femoral muscles (quadriceps) during isometric knee extension before versus after intervention (1.58 [1.29-1.88] versus 1.78 [1.34-1.97] Nm/kg, $P=0.004)$. In contrast, there was no significant change in the strength of the femoral muscles (hamstrings) during isometric knee flexion $(0.64$ [0.57-0.73] versus $0.70[0.62-0.79] \mathrm{Nm} /$ $\mathrm{kg}, P=0.18)$. Magnetic resonance imaging analysis of the quadriceps (cross-sectional area) showed a significant increase (55.2 [49.0-64.4] versus 56.9 [46.6-66.7] $\mathrm{cm}^{2}$, $P=0.020$, Figure $2 \mathrm{~B})$. However, there was no significant 
change in the strength of the hamstrings (22.2 [19.1-28.7] versus $\left.21.9[19.4-25.9] \mathrm{cm}^{2}, P=0.43\right)$.

\section{Proton magnetic resonance spectroscopy}

The participants, ie, obese patients with NAFLD, showed significantly higher levels of both IMCL and EMCL compared with normal subjects at both baseline and after 12 weeks of $\mathrm{AT}$ intervention (Figure 2C). The AT intervention reduced the level of IMCL in obese patients (4.59 [3.00-6.38] versus $3.38[2.97-5.40] \mathrm{mmol} / \mathrm{kg}, P=0.044)$ but not of EMCL $(19.00$ [10.14-21.88] versus 14.66 [8.98-22.50] mmol $/ \mathrm{kg}, P=0.78$, Figure 2C). In addition, obese patients with NAFLD showed significantly higher intrahepatic lipid levels than normal subjects at both baseline and after 12 weeks of AT intervention (Figure 2D). Obese patients with NAFLD showed significant reduction after AT intervention $(0.138$ [0.054-0.253] versus 0.110 [0.044-0.209], $P=0.007$, Figure 2D).

\section{Measurement of liver steatosis and stiffness by Fibroscan 502}

The participants showed a significant reduction in CAP, reflecting the degree of liver steatosis after AT intervention (310 [245-316] versus 283 [197-306] dB/m, $P=0.009$, Figure $2 \mathrm{E}$ ) and a declining trend in the stiffness value (6.2 [4.9-8.9] versus 5.0 [4.3-8.9] kPa, $P=0.083$, Figure 2F). However, compared with normal subjects, the participants had high CAP and stiffness values at both baseline and after 12 weeks of AT intervention, respectively (Figure 2E and F).

\section{Blood analysis}

Of the 16 parameters in the liver function tests, the baseline level of 13 , except for platelets, low-density lipoprotein cholesterol, and hyaluronan, a showed a significant difference compared with normal subjects $(P<0.05)$. However, three parameters, including ferritin, high-sensitivity C-reactive protein, and free fatty acids, showed no significant difference compared with normal subjects after the AT intervention. Liver function tests significant improvements in ALT $(-47.6 \%)$, gamma glutamyl transpeptidase $(-7.1 \%)$, free fatty acids $(-16.4 \%)$, and total cholesterol $(-5.1 \%)$ after the intervention, as shown in Table 2.

With regard to adipokines, the leptin, TNF- $\alpha$, and interleukin-6 levels were significantly higher and the adiponectin level was significantly lower in the study subjects than in the controls (Figure 3A). The participants showed a significant improvement in levels of leptin (31.9 [16.9-53.5] versus 28.8 [15.3-36.3] ng/mL, $P=0.031$ ), adiponectin (3.69 [2.72-6.24] versus 4.01 [3.29-6.55] ng/mL, $P=0.022)$, TNF- $\alpha(1.45$ [0.53-2.11] versus 1.19 [0.34-1.82] pg/mL, $P=0.012)$, and interleukin-6 (2.05 [1.81-3.08] versus $1.50[1.24-2.32] \mathrm{pg} / \mathrm{mL}$, $P=0.011$, Figure $3 \mathrm{~A})$. In contrast, no improvement was observed in the levels of high-sensitivity C-reactive protein (an inflammatory marker) and thiobarbituric acid reactive substances (an oxidative stress marker, Figure 3B).

\section{Health-related quality of life}

Eight subscales were used to measure the effect of the AT program on quality of life ie, physical functioning, physical role, bodily pain, emotional role, mental health, general health perception, vitality, and social functioning. Of these, significant improvements were observed in levels of physical functioning (41.6 [32.6-50.6] versus 48.8 [39.8-54.2], $P=0.009)$, physical role (50.8 [42.4-55.7] versus 55.7 [52.4-55.7], $P=0.042)$, general health perception (38.4 [35.1-49.5] versus 46.9 [38.9-49.5], $P=0.011)$, and social functioning (53.8 [37.7-57.0] versus 57.0 [50.6-57.0], $P=0.036$, Figure 4).

\section{Discussion}

The important finding of this pilot study is that AT attenuates body adiposity in obese patients with NAFLD. A significant reduction in hepatic fat content as well as IMCL was also achieved with a program consisting of 20-minute AT sessions twice a week for 12 weeks (Figure 2), along with improvement in liver function test abnormalities (Table 2). In addition, the results of the health-related quality of life survey highlighted improvements in both physical and mental parameters (Figure 4). Overall, these results indicate that AT is particularly effective in the management of NAFLD in obese patients, with positive effects on their mental and physical health status.

It has been suggested that resistance training is not sufficiently effective for weight loss. ${ }^{8,21}$ Furthermore, reports suggest that the reduction in body fat achieved by resistance training is minimal ${ }^{22-24}$ or nil. ${ }^{25}$ Consistent with these studies, we found that AT only modestly decreased body weight as well as body fat mass and visceral fat area in obese subjects with NAFLD (Table 1). Induction of adipose tissue loss by AT through an increase in metabolic rate has been reported previously. ${ }^{12}$ One possibility is that myogenesis induced by AT leads to recruitment of a large number of muscle fibers, resulting in an increase in the metabolic potential to burn fat. Another possibility is that of activation of the sympathetic nervous system by $\mathrm{AT},{ }^{26}$ which induces lipolysis in white adipose tissue, resulting in reduction of body fat. ${ }^{13}$ On the other hand, skeletal muscle mass, EE, and the nonprotein 
A

(ng/mL)

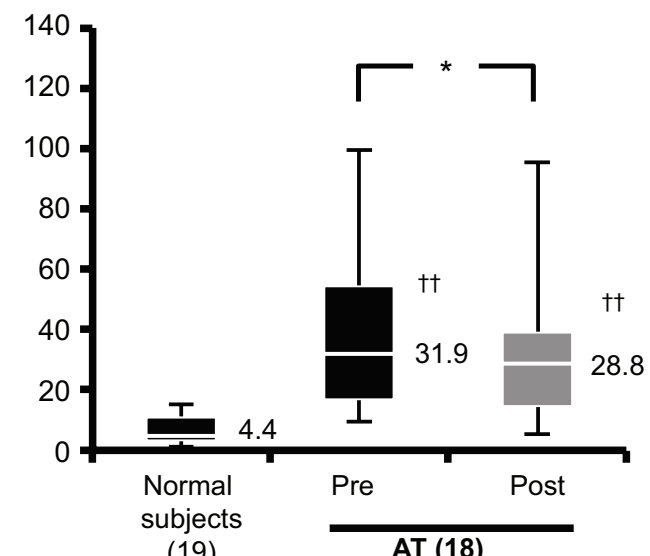

(19)

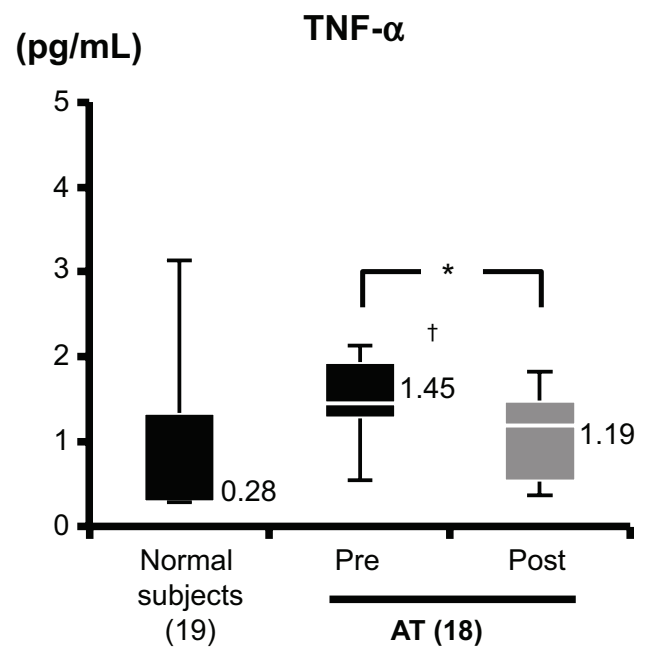

B



Adiponectin

\section{( $\mu \mathrm{g} / \mathrm{mL})$}

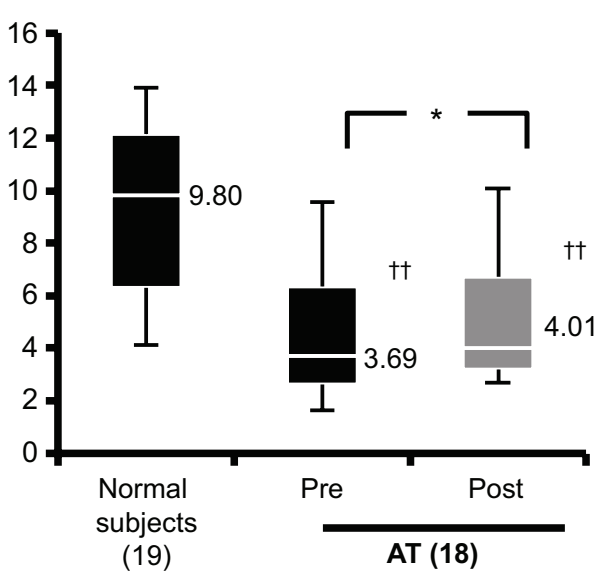

$(\mathrm{pg} / \mathrm{mL}) \quad \mathrm{IL}-6$

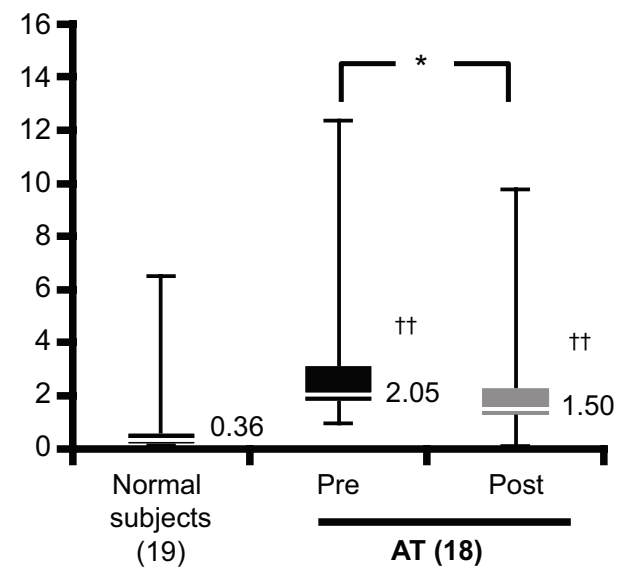

TBARS

( $\mu$ moles/L)

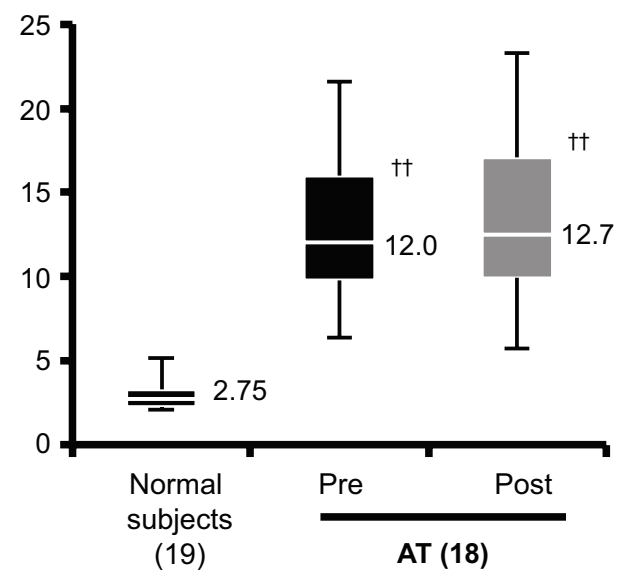

Figure 3 Effects of AT on serum levels of leptin, adiponectin, TNF- $\alpha$, IL-6 (A), and hs-CRP and TBARS (B) in obese patients with nonalcoholic fatty liver disease. Notes: $* P<0.05$, significant difference between the baseline and week 12 . ${ }^{\dagger} P<0.05$, ${ }^{\dagger} P<0.0$ I, significant difference between groups (normal subject group versus AT group pre and post). The lower edge, midline, and upper edge of each box represent the 25 th percentile, median, and 75 th percentile scores, respectively. The line from each box extends to the minimum and maximum score.

Abbreviations: AT, acceleration training; TNF- $\alpha$, tumor necrosis factor-alpha; IL-6, interleukin-6; hsCRP, high-sensitivity C-reactive protein; TBARS, thiobarbituric acid reactive substances. 


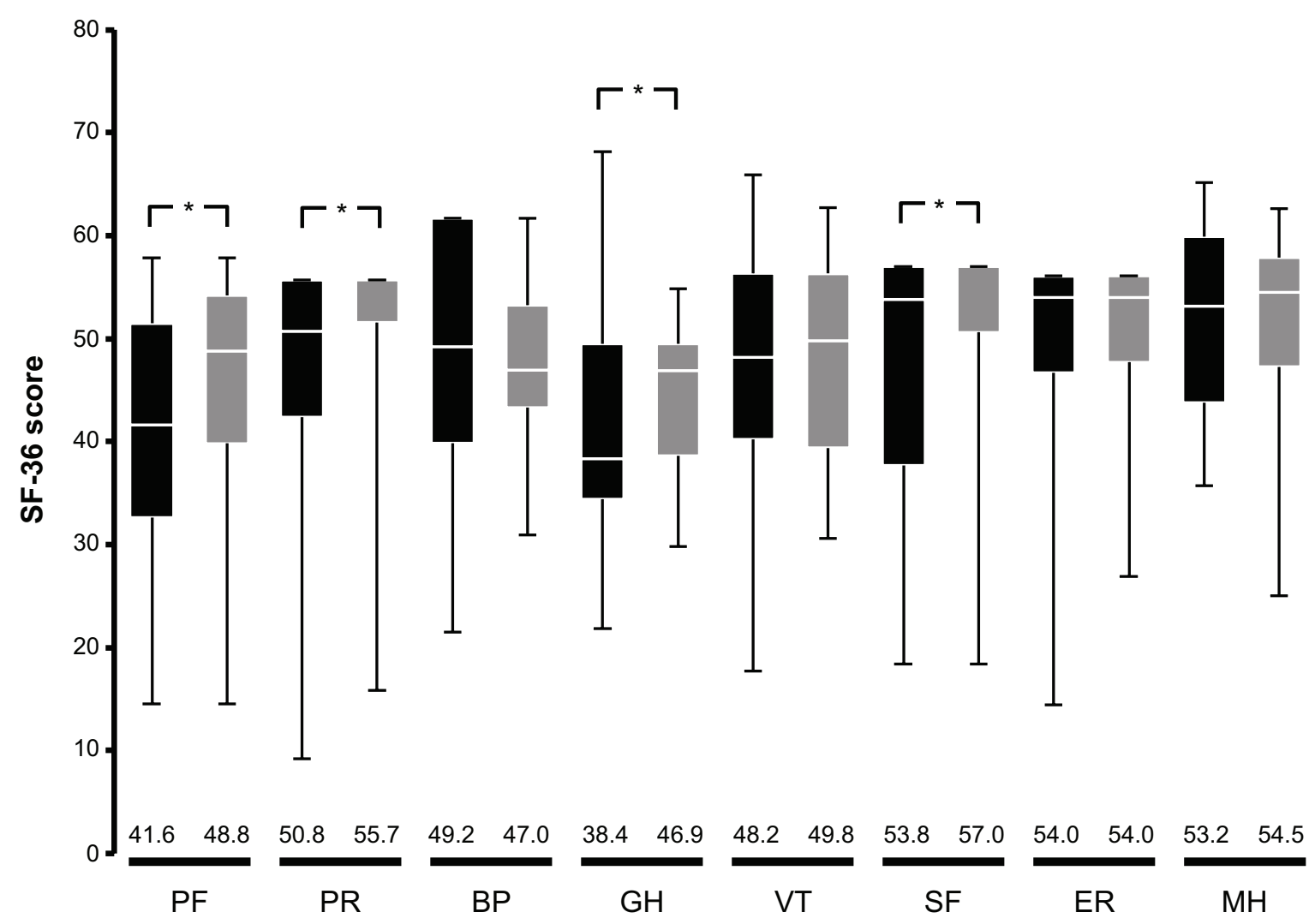

Figure 4 Changes in SF-36 scores prior to and after acceleration training in obese patients with nonalcoholic fatty liver disease. Notes: $* P<0.05$, significant difference between baseline and week 12 .

Abbreviations: BP, bodily pain; GH, general health perception; SF-36, Short Form Health Survey; ER, emotional role; MH, mental health; PF, physical functioning; PR, physical role; SF, social functioning; VT, vitality.

respiratory quotient was not affected (Table 1). Recently, skeletal muscle has been identified as not only a component of the locomotor system but also a metabolic organ engaged in glucose and fatty acid metabolism similar to the liver and adipose tissue. ${ }^{27}$ It is therefore remarkably advantageous that AT can maintain skeletal muscle mass. In addition, our study showed that AT increased the strength of skeletal muscles (Figure 2). According to Bonner Physical Therapy in the USA, an AT program for 6 weeks results in an increase in resting energy metabolism by $18 \%$ and changes in body composition, such as reduced body fat and increased fat-free mass. ${ }^{28}$ Collectively, these reports and this study suggest that AT can be utilized to reduce body fat and/or visceral fat while maintaining skeletal muscle mass as well as improving its physical function.

The molecular mechanism by which AT attenuates body adiposity (Table 1) and also reduces hepatic fat content as well as IMCL in obese patients with NAFLD (Figure 2) remains unknown. Resistance training is hypothesized to reduce the amount of hepatic fat via a mechanism involving insulin sensitivity and fatty acid metabolism. ${ }^{6}$ In NAFLD, the level of intrahepatic lipid increases via induction of de novo lipogenesis, resulting from induced expression and activation of transcription factors, such as sterol regulatory element-binding protein-1c and carbohydrate response element-binding protein, in response to increased insulin levels. ${ }^{29}$ In addition, increased levels of triglyceride intermediates inhibit insulin-stimulated glucose uptake, suggesting a vicious cycle whereby inhibition of insulin action by high intrahepatic lipid levels in the liver triggers a further increase in intrahepatic lipid levels. ${ }^{29}$

It has been reported that resistance training restores insulin sensitivity and increases systemic glucose metabolism through induction and activation of skeletal muscle GLUT4, followed by an increase in glucose uptake by skeletal muscle and induction of glycogen synthesis. ${ }^{11,30}$ Resistance training has also been shown to reduce hepatic fatty acid uptake and increase the utilization of fatty acids by skeletal muscle via enhanced myogenesis, leading to a reduction of hepatic fat content. ${ }^{6}$ The effect of WBV training on the endocrine system in normal male subjects was investigated by Di Loreto et al under the hypothesis that application of vibrations would 
be effective in the treatment of obesity. ${ }^{31}$ They showed that WBV training improved glucose utilization by increasing the consumption of circulating plasma glucose for muscle contraction, indicating that WBV training improves insulin sensitivity. ${ }^{31}$ Thus, long-term WBV training is believed to attenuate insulin resistance in obese and diabetic patients by repeated vibratory stimulation of skeletal muscles, subsequently leading to muscle contractions. A similar mechanism is assumed to be relevant in AT.

Another important finding of this study is that AT alleviates adipokine imbalance (Figure 3 ). In recent years, adipose tissue has been recognized as an endocrine organ that secretes various biologically active substances such as adipokines. ${ }^{32}$ Aberrant expression and secretion of adipokines (adipokine imbalance) is induced by hypertrophy of adipocytes, a condition associated with obesity, resulting in the secretion of inflammatory cytokines (such as TNF- $\alpha$ and interleukin-6) or metabolic products of adipose tissue (such as leptin and free fatty acids). In contrast, production of adiponectin, a member of the "good" adipokine group, is reduced. This adipokine imbalance largely affects the development and progression of NAFLD, which is a primary causative factor in insulin resistance. ${ }^{33}$ The fall in serum levels of leptin, TNF- $\alpha$, interleukin- 6 , and free fatty acids, and the increase in serum levels of adiponectin elicited by AT are assumed to result from a decrease in body fat and changes in the cellular characteristics of adipocytes. However, the precise underlying mechanisms are not clear. Leptin acts through the sympathetic nervous system to induce lipolysis. ${ }^{34}$ Meanwhile, adiponectin induces $\beta$-oxidation of fatty acids. ${ }^{33,35}$ Improvement of liver function test abnormalities by AT (Table 2) is speculated to be the consequence of restoration of insulin sensitivity and reduction of aberrant fat accumulation in the liver through the resolution of adipokine imbalance, thereby alleviating inflammation and oxidative stress conditions in the liver. Changes to the degree of liver steatosis and stiffness analyzed using Fibroscan 502 also confirmed improvements in the pathogenic conditions of the liver through AT in obese patients with NAFLD (Figure 2).

In terms of future prospects, the unsuitability of weightoriented aerobic training for obese people with NAFLD because of the difficulty in maintaining weight loss necessitates the development of alternative strategies such as resistance training. A systematic review and meta-analysis of the evidence from prospective studies has shown that increased levels of physical activity significantly reduce serum aminotransferase levels and hepatic steatosis in patients with NAFLD regardless of the degree of weight loss. ${ }^{36}$ Therefore, clinicians need to consider the benefits associated with increased physical activity outside the scope of weight loss and take into account the long-term sustainability of a weight loss strategy as well as the feasibility of particular physical activities tailored for each patient.

In conclusion, the results of this pilot study suggest that AT is effective in improving physical function, ameliorating hepatic steatosis and liver function test abnormalities, and improving or maintaining the quality of life in obese patients with NAFLD. Thus, AT is a promising new approach that is likely to be employed in therapeutic exercise interventions. The major limitation of this study is the absence of an appropriate control group, which is likely to lead to spurious causality and bias. In the future, a large-scale randomized study should be conducted to investigate the effectiveness of AT in the management of NAFLD.

\section{Acknowledgment}

This work was supported in part by Grants-in-Aid for Scientific Research from the Ministry of Education, Culture, Sports, Science and Technology, Japan (24390488, 25282172, 25560364, 26670109).

\section{Disclosure}

The authors have no financial relationships to disclose with regard to this study.

\section{References}

1. Eguchi E, Iso H, Tanabe N, et al. Healthy lifestyle behaviours and cardiovascular mortality among Japanese men and women: the Japan collaborative cohort study. Eur Heart J. 2012;33(4):467-477.

2. Survey in Japan Society of Ningen. Japan Society of Ningen Doc. Available from: http://www.ningen-dock.jp/wp/common/data/other/release/ dock-genkyou_h24.pdf. Accessed September 20, 2014.

3. Targher G. Non-alcoholic fatty liver disease, the metabolic syndrome and the risk of cardiovascular disease: the plot thickens. Diabet Med. 2007;24(1):1-6.

4. Harrison SA, Day CP. Benefits of lifestyle modification in NAFLD. Gut. 2007;56(12):1760-1709.

5. Johnson NA, George J. Fitness versus fatness: moving beyond weight loss in nonalcoholic fatty liver disease. Hepatology. 2010;52(1):370-381.

6. Hallsworth K, Fattakhova G, Hollingsworth KG, et al. Resistance exercise reduces liver fat and its mediators in non-alcoholic fatty liver disease independent of weight loss. Gut. 2011;60(9):1278-1283.

7. Bacchi E, Negri C, Targher G, et al. Both resistance training and aerobic training reduce hepatic fat content in type 2 diabetic subjects with nonalcoholic fatty liver disease (the RAED2 Randomized Trial). Hepatology. 2013;58(4):1287-1295.

8. Donnelly JE, Blair SN, Jakicic JM, Manore MM, Rankin JW, Smith BK. American College of Sports Medicine Position Stand. Appropriate physical activity intervention strategies for weight loss and prevention of weight regain for adults. Med Sci Sports Exerc. 2009;41(2): 459-471.

9. Dolezal BA, Potteiger JA. Concurrent resistance and endurance training influence basal metabolic rate in nondieting individuals. $J$ Appl Physiol. 1985;85(2):695-700. 
10. Rittweger J. Vibration as an exercise modality: how it may work, and what its potential might be. Eur J Appl Physiol. 2010;108(5):877-904.

11. Holten MK, Zacho M, Gaster M, Juel C, Wojtaszewski JF, Dela F. Strength training increases insulin-mediated glucose uptake, GLUT4 content, and insulin signaling in skeletal muscle in patients with type 2 diabetes. Diabetes. 2004;53(2):294-305.

12. Vissers D, Verrijken A, Mertens I, et al. Effect of long-term whole body vibration training on visceral adipose tissue: a preliminary report. Obes Facts. 2010;3(2):93-100.

13. So R, Eto M, Tsujimoto T, Tanaka K. Acceleration training for improving physical fitness and weight loss in obese women. Obes Res Clin Pract. 2014;8(3):e238-e248.

14. Farrell GC, Chitturi S, Lau GK, Sollano JD. Guidelines for the assessment and management of non-alcoholic fatty liver disease in the AsiaPacific region: executive summary. J Gastroenterol Hepatol. 2007;22(6): 775-777.

15. American Gastroenterological Association. American Gastroenterological Association medical position statement: nonalcoholic fatty liver disease. Gastroenterology. 2002;123(5):1702-1704.

16. Saadeh S, Younossi ZM, Remer EM, et al. The utility of radiological imaging in nonalcoholic fatty liver disease. Gastroenterology. 2002; 123(3):745-750

17. Matthews DR, Hosker JP, Rudenski AS, Naylor BA, Treacher DF, Turner RC. Homeostasis model assessment: insulin resistance and betacell function from fasting plasma glucose and insulin concentrations in man. Diabetologia. 1985;28(7):412-419.

18. Sandrin L, Fourquet B, Hasquenoph JM, et al. Transient elastography: a new noninvasive method for assessment of hepatic fibrosis. Ultrasound Med Biol. 2003;29(12):1705-1713.

19. Sasso M, Beaugrand M, de Ledinghen V, et al. Controlled attenuation parameter (CAP): a novel VCTE guided ultrasonic attenuation measurement for the evaluation of hepatic steatosis: preliminary study and validation in a cohort of patients with chronic liver disease from various causes. Ultrasound Med Biol. 2010;36(11):1825-1835.

20. Ware JE Jr, Gandek B. Overview of the SF-36 Health Survey and the International Quality of Life Assessment (IQOLA) Project. J Clin Epidemiol. 1998;51(11):903-912.

21. Borg P, Kukkonen-Harjula K, Fogelholm M, Pasanen M. Effects of walking or resistance training on weight loss maintenance in obese, middle-aged men: a randomized trial. Int J Obes Relat Metab Disord. 2002;26(5):676-683.

22. Schmitz KH, Jensen MD, Kugler KC, Jeffery RW, Leon AS. Strength training for obesity prevention in midlife women. Int J Obes Relat Metab Disord. 2003;27(3):326-333.

23. Lee S, Bacha F, Hannon T, Kuk JL, Boesch C, Arslanian S. Effects of aerobic versus resistance exercise without caloric restriction on abdominal fat, intrahepatic lipid, and insulin sensitivity in obese adolescent boys: a randomized, controlled trial. Diabetes. 2012;61(11):2787-2795.
24. Hunter GR, Bryan DR, Wetzstein CJ, Zuckerman PA, Bamman MM. Resistance training and intra-abdominal adipose tissue in older men and women. Med Sci Sports Exerc. 2002;34(6):1023-1028.

25. Polak J, Moro C, Klimcakova E, et al. Dynamic strength training improves insulin sensitivity and functional balance between adrenergic alpha $2 \mathrm{~A}$ and beta pathways in subcutaneous adipose tissue of obese subjects. Diabetologia. 2005;48(12):2631-2640.

26. Ando H, Noguchi R. Dependence of palmar sweating response and central nervous system activity on the frequency of whole-body vibration. Scand J Work Environ Health. 2003;29(3):216-219.

27. Jensen J, Rustad PI, Kolnes AJ, Lai YC. The role of skeletal muscle glycogen breakdown for regulation of insulin sensitivity by exercise. Front Physiol. 2011;2:112. Available from: http://journal.frontiersin.org/ Journal/10.3389/fphys.2011.00112/full. Accessed September 20, 2014.

28. Van der Meer G, Zeinstra E, Temperlaars J, Hopson S. Handbook of Acceleration Training: Science, Principles, and Benefits. Northbrook, IL, USA: Power Plate International; 2007.

29. Fabbrini E, Sullivan S, Klein S. Obesity and nonalcoholic fatty liver disease: biochemical, metabolic, and clinical implications. Hepatology. 2010;51(2):679-689.

30. Holten MK, Zacho M, Gaster M, Juel C, Wojtaszewski JF, Dela F. Strength training increases insulin-mediated glucose uptake, GLUT4 content, and insulin signaling in skeletal muscle in patients with type 2 diabetes. Diabetes. 2004;53(2):294-305.

31. Di Loreto C, Ranchelli A, Lucidi P, et al. Effects of whole-body vibration exercise on the endocrine system of healthy men. $J$ Endocrinol Invest. 2004;27(4):323-327.

32. Tilg H, Moschen AR. Adipocytokines: mediators linking adipose tissue, inflammation and immunity. Nat Rev Immunol. 2006;6(10): 772-783.

33. Yamauchi T, Kamon J, Minokoshi Y, et al. Adiponectin stimulates glucose utilization and fatty-acid oxidation by activating AMP-activated protein kinase. Nat Med. 2002;8(11):1288-1295.

34. Pinkney JH, Coppack SW, Mohamed-Ali V. Effect of isoprenaline on plasma leptin and lipolysis in humans. Clin Endocrinol. 1998; 48(4):407-411.

35. Yoon MJ, Lee GY, Chung JJ, Ahn YH, Hong SH, Kim JB. Adiponectin increases fatty acid oxidation in skeletal muscle cells by sequential activation of AMP-activated protein kinase, p38 mitogen-activated protein kinase, and peroxisome proliferator-activated receptor alpha Diabetes. 2006;55(9):2562-2570.

36. Keating SE, Hackett DA, George J, Johnson NA. Exercise and nonalcoholic fatty liver disease: a systematic review and meta-analysis. J Hepatol. 2012;57(1):157-166. 


\section{Supplementary material}


Figure SI The acceleration training program conducted in this study.

\section{Publish your work in this journal}

Therapeutics and Clinical Risk Management is an international, peerreviewed journal of clinical therapeutics and risk management, focusing on concise rapid reporting of clinical studies in all therapeutic areas, outcomes, safety, and programs for the effective, safe, and sustained use of medicines. This journal is indexed on PubMed Central, CAS,
EMBase, Scopus and the Elsevier Bibliographic databases. The manuscript management system is completely online and includes a very quick and fair peer-review system, which is all easy to use. Visit http://www.dovepress.com/testimonials.php to read real quotes from published authors. 